\title{
Methods for Development of Microsatellite Markers: An Overview
}

\author{
Siju SENAN, Dhanya KIZHAKAYIL, Bhaskaran SASIKUMAR, \\ Thotten Elampilay SHEEJA* \\ Division of Crop Improvement and Biotechnology, Indian Institute of Spices Research, Calicut 673012, Kerala, India; \\ teshee@rediffmail.com (* corresponding author) \\ Telephone: +91-495-2731410; Fax: +91-495-2731187
}

\begin{abstract}
Microsatellite or Simple Sequence Repeat (SSR) markers have evolved to the status of a most versatile and popular genetic marker in a ubiquity of plant systems. Due to their co-dominant, hyper-variable and multiallelic nature, they are the prominent markers of choice for fingerprinting, conservation genetics, plant breeding and phylogenetic studies. Despite its development of a new set of SSR markers for a species remained time consuming and expensive for many years. However, with the recent advancement in genomics, new strategies/protocols are now available for the generation of SSR markers. This review presents an overview on microsatellite markers with a special emphasis on the various strategies used for the development of microsatellite markers.
\end{abstract}

Keywords: Chloroplast SSR, EST-SSR, Genetic diversity, Genomic SSR, Microsatellite isolation strategies, Simple Sequence Repeats

\section{Introduction}

Microsatellites are stretches of DNA consisting of tandemly arranged units of 1-6 bp (Gupta et al., 1996; Thiel et al., 2003), characterised by their co-dominant inheritance, wide genomic distribution, hyper variable and multiallelic nature (Powell et al., 1996; Parida et al., 2009). They are also termed as simple sequences (Tautz 1989), Short Tandem Repeats (STRs) (Edwards et al., 1991) and Simple Sequence Repeats (SSRs) (Jacob et al., 1991). The existence of microsatellites in a wide range of evolutionarily diverse eukaryotic genomes (from yeasts to humans) was first documented by Hamada et al. (1982). Tautz and Renz (1984) further confirmed their abundance in five phylogenetically distinct eukaryotes including human, Drosophila, yeast, sea urchin and Stylonychia. The polymorphic nature of microsatellite was revealed by Litt and Luty (1989) by amplifying $(\mathrm{TG})_{\mathrm{n}}$ microsatellites in the human cardiac actin gene and detecting allelic variants in unrelated individuals. Weber and May (1989) also reported the successful amplification and polymorphic nature of SSR loci. The genesis of these repeats occur primarily due to slippedstrand mispairing (Levinson and Gutman, 1987) and subsequent errors during DNA replication/ repair/ recombination (Schlotterer and Tautz, 1992; Katti et al., 2001), nucleotide composition of repeat motifs (Katti et al., 2001) or unequal crossing-over between sister chromatids (Innan et al., 1997). However, a minimum size threshold (eight nucleotides or more) is crucial for the dynamic slippage mutation of microsatellites
(Messier et al., 1996; Rose and Falush, 1998).

Microsatellites are ubiquitous in the coding and noncoding regions (Tautz and Renz, 1984; Gupta et al., 1996; Toth et al., 2000) with a higher density of simple sequence motifs in the noncoding regions of eukaryotes (Hancock, 1995; Li et al., 2002). In plants, SSRs are much more abundant and preferentially associated within untranslated regions (UTRs) of the transcribed regions (Morgante et al., 2002). The frequency of repeats decrease exponentially with repeat length (Metzgar et al., 2000; Katti et al., 2001) with their informativeness correlating to the repeat length (Lagercrantz et al., 1993). This might be due to the fact that longer microsatellites exhibits higher mutation rates than shorter ones (McConnell et al., 2007).

Among the different types of SSRs, dinucleotide, trinucleotide and tetranucleotide repeats are most frequently used in molecular genetic studies (Selkoe and Toonen, 2006). Dinucleotide repeats constitute the majority of microsatellites reported from many species and are less frequent in coding region than in non-coding region ( $\mathrm{Li}$ et al., 2002). In contrary, trinucleotides are more abundant in the coding regions of the genome (Toth et al., 2000). In plants, monocots are enriched with GC-rich trinucleotide repeats than dicots (Morgante et al., 2002). The relative abundance of trinucleotide repeats in the protein-coding regions of all taxa is attributed to the negative selection against frameshift mutations occurring in coding regions and probably positive selection for specific single amino-acid stretches (Metzgar et al., 2000). 


\section{Types of microsatellites}

Based on occurrence and source for development

Three types of SSRs:

a. Genomic or nuclear microsatellites (gSSRs) microsatellites isolated from the nuclear genome (genomic DNA of an organism with or without the construction of genomic DNA library).

b. EST or genic microsatellites (EST-SSRs) microsatellites developed by data-mining or exploiting EST sequences deposited in public databases.

c. Organellar microsatellites [chloroplast SSRs (cpSSRs) and mitochondrial SSRs (mtSSRs)] microsatellites developed from the chloroplast or mitochondrial genome of an organism.

\section{Based on the type of repeat sequence}

Four types of SSRs (Oliveira et al., 2006):

a. Perfect microsatellite- the repeat sequence is continuous and is not interrupted by any base not belonging to the motif [e.g. AGAGAGAGAGAG or $(\mathrm{AG})_{6}$

b. Imperfect microsatellite- a pair of bases is present between the repeat motif that does not match the motif sequence [e.g. AGAGAGAGAGCTAGAGAG or $\left.(\mathrm{AG})_{5} \mathrm{CT}(\mathrm{AG})_{3}\right]$.

c. Interrupted microsatellite- a small sequence within the repeated sequence that does not match the motif sequence [e.g. AGAGAGAGCGTGAGAGAGAG or $\left.(\mathrm{AG})_{4} \mathrm{CGTG}(\mathrm{AG})_{4}\right]$.

d. Compound/ composite microsatellite- two adjacent distinctive repeats present within the sequence [e.g. AGAGAGAGAGTCTCTCTC or $\left.(\mathrm{AG})_{5}(\mathrm{TC})_{4}\right]$.

Based on the length of repeat motif

Two types of SSRs (Temnykh et al., 2001):

a. Class I microsatellites- perfect SSRs of $\geq 20$ nucleotides in length.

b. Class II microsatellites- perfect SSRs of $\geq 12$ nucleotides and $\leq 20$ nucleotides in length.

\section{Microsatellites and their influence on molecular functions}

The presence of SSRs in the coding regions lead to the appearance of repetitive patterns in the aminoacid sequences (Katti et al., 2001) and thus involve in regulating gene expression or molecular functions. Occurrence of SSRs in the promoter region influences transcriptional activity (Kashi et al., 1997), whereas their presence in non coding regions influences gene regulation, transcription (Martin et al., 2004; Lawson and Zhang, 2006) and recombination events (Bagshaw et al., 2008). The over-representation of CT/GA and CTT/GAA repeats in the 5'-flanks of Arabidopsis thaliana suggest their potential involvement in regulating gene expression (Zhang et al., 2004). The (GA)n repeats in promoters govern the regulation of certain plant genes (Meister et al., 2004) and exhibit protein-binding affinity
(Kooiker et al., 2005). The CT/GA repeat variation in the 5' UTR of the waxy gene is correlated with amylose content in rice (Bao et al., 2002). In maize, presence of $(\mathrm{CCG})_{\mathrm{n}}$ in the 5' UTR of ribosomal protein genes regulate fertilization (Dresselhaus et al., 1999). Polystretches of glutamine (Gerber et al., 1994) and proline (Perutz et al., 1994) encoded by rapidly evolving repeats are known to modulate the activity of transcription factors. Similarly, the presence of the trinucleotide repeats like $(\mathrm{GAA})_{\mathrm{n}}$ within $5^{\prime} \mathrm{UTR}$ of $n t p 303$ regulate transcription and translation (Hulzink et al., 2002).

\section{General advantages and disadvantages of microsatellite markers}

Though microsatellite markers are considered to be robust, there are also advantages and disadvantages associated with the level of polymorphism and mode of application.

Microsatellites are more variable and informative than RFLP, RAPD (He et al., 2003) and AFLPs (Lee et al., 2004). The technique is PCR-based, thus require only low quantities of template DNA (Kumar et al., 2009; Wolko et al., 2010). The application of lengthy primers and high annealing temperatures during genotyping enhances reproducibility. The ability to use more than one set of optimized SSR markers in a single reaction (multiplexing of markers) significantly reduces the analytical costs involved in genome analysis. They are also useful for parentage analysis and for estimating the degree of relatedness of individuals or groups. Multiallellic microsatellites are considered to be the best marker system for the detection of intervarietal polymorphisms (Stepien et al., 2007). They offer wide applications in the preparation of genome-wide genetic maps and comparative mapping.

However, the de novo development of SSR marker is expensive, laborious and time-consuming (Zane et al., 2002: Squirrell et al., 2003; Thiel et al., 2003). Low frequency of SSRs in plants also hinders the large scale isolation of SSRs (Powell et al., 1996). Moreover, SSR markers developed for one species generally exhibit less transferability across same or different taxa which necessitate the development of species specific markers (Roa et al., 2000; Kindiger, 2006). Another important problem associated with microsatellites is the occurrence of null alleles. The potential cause is poor primer annealing caused by nucleotide sequence divergence, inconsistent DNA template quality or low template quantity (Ellegren, 2004) or mutations /indels in the primer binding sites (Pemberton et al., 1995). This leads to complications in the determination of allelic and genotypic frequencies and an underestimation of heterozygosity (Kumar et al., 2009). Homoplasy is another problem when applying microsatellites as a reliable tool for phylogenetic analysis because alleles considered to be identical in state are not necessarily identical by descent (Estoup et al., 2002).

\section{Strategies for microsatellite development}

For generating a new set of polymorphic SSR marker for species, microsatellite repeats should be isolated or identified along with sufficient flanking nucleotide sequence information to facilitate primer designing. The 
PCR conditions need to be optimized and the primers need to be screened in a set of related and non related individuals for estimating their polymorphic potential.

The protocol for the de novo isolation of microsatellite markers was first described by Rassmann $e t$ al. (1991), who identified SSR-containing clones by colony hybridization with SSR probes. However it turned out to be laborious and expensive for species with low frequency of SSRs. The strategies used for the effective isolation of SSR loci were reviewed earlier by Zane et al. (2002) and Kalia et al. (2011). However, with the advancement in genomics, availability of new molecular tools and sequencing platforms for exploring genomic information, several new protocols were developed in the recent years. A general outline on the development of microsatellite markers is summarized (Fig.1) and could be achieved either by:

a) constructing and screening SSR enriched/non enriched genomic libraries or by utilizing the products generated by other molecular markers or by the application of next-generation sequencing systems (gSSRs).

b) exploiting the EST/chloroplast sequences deposited in the public domain (EST-SSRs/ cpSSRs) or sequencing PCR products generated by "consensus/universal chloroplast primers" (cpSSRs).

d) testing the amplification potential of SSR markers developed in other related species (transferability/ crossspecies amplification).

\section{Genomic SSR markers}

\section{Development of microsatellite markers from SSR-enriched genomic DNA libraries}

Briefly, the methods used for isolating SSRs by constructing genomic libraries can be grouped into two categories.

i) Selective hybridization methods

These methods facilitate the selective isolation of microsatellite containing DNA portions of the genome by hybridisation with repeat-specific probes. These protocols generally involve the fragmentation of DNA either by sonication (Karagyozov et al., 1993; Kandpal et al., 1994; Geng et al., 2010) or restriction enzymes (Brown et al., 1995; Chen et al., 1995; Edwards et al., 1996; Prochazka., 1996; Refseth et al., 1997; Fischer and Bachmann, 1998; Hamilton et al., 1999; Glenn and Schable, 2005; Nunome et al., 2006) or nebulisation (Kumpatla et al., 2004; Connell et al., 1998) and its subsequent ligation to a known sequence (linker or adaptors) or directly to a vector. DNA is then denatured and subjected to enrichment by hybridization with

a) biotinylated oligos followed by capture of biotinylated hybrids (oligo bound DNA fragments) in vectrex-avidin matrix (Kandpal et al., 1994) or

b) oligonucleotides bound to nylon membrane (Karagyozov et al., 1993; Chen et al., 1995; Edwards et al., 1996) or

c) 5' biotinylated repeat oligos and subsequent capture of biotinylated hybrids by streptavidin coated magnetic beads (Brown et al., 1995; Refseth et al., 1997; Fischer and
Bachmann, 1998; Connell et al., 1998; Hamilton et al., 1999; Kumpatla et al., 2004; Dixit et al., 2005; Glenn and Schable, 2005; Nunome et al., 2006; Geng et al., 2010) or

d) 'biotinylated SSR probe-streptavidin coated magnetic bead complex' ('Triplex affinity capture' protocol; White and Powell, 1997).

The enriched DNA fragments were then amplified, either cloned and sequenced or sequenced directly and searched for the presence of SSR motifs. The efficiency of this approach entirely depends on the specific binding of streptavidin coated beads to the biotin labelled DNA fragments harbouring SSRs.

ii) Primer extension methods

These methods permit selective amplification of microsatellite containing genomic DNA using SSR specific primers (Ostrander et al., 1992; Pandolfo, 1992; Robic et al., 1994; Paetkau, 1999). This procedure (Ostrander et al., 1992; Paetkau, 1999) relies on the construction of a primary genomic library in phagemid vector to recover the library as single stranded DNA (ssDNA) which is subjected to primer extension using repeat specific non-biotinylated oligos (Ostrander et al., 1992) or $5^{\prime}$ biotinylated oligos (Paetkau, 1999). These primer extension steps that selectively generate double stranded products only from vectors containing the desired repeats were transformed into $E$. coli cells (Ostrander et al., 1992). The primer extended products generated using 5' biotinylated oligos (Paetkau, 1999) were selectively captured using streptavidin coated magnetic beads and converted to double stranded DNA (dsDNA) by a second round of primer extension for transformation.

Another protocol based on primer extension (Pandolfo, 1992) involved the ligation of a vectorette (linker containing a non-complementary region) to restricted YAC (Yeast Artificial Chromosome) DNA. The vectorrete-ligated DNA was amplified (using repeat specific primer along with a universal vector primer) and the products were cloned and sequenced for detecting SSRs. A modified version of this approach termed as the 'SLiM-PCR' (Subcloning Ligation Mixture-PCR; Robic et al., 1994) allowed the sequencing of flanking regions of a microsatellite from a cosmid clone using a fluorescent automatic sequencing method without subcloning.

Among the different enrichment protocols available, selective hybridization capture is the predominantly used strategy as it allows enrichment and selection prior to cloning thereby providing a faster and easier method to work with multiple samples (Glenn and Schable, 2005). Moreover, it is relatively simple, reproducible and cost effective approach for isolating microsatellites from diverse plant species with higher efficiency (Kalia et al., 2011).

\section{Development of microsatellite markers from non-enriched} genomic DNA libraries

In this method, the genomic DNA was restricted, ligated into suitable vectors and transformed to generate a non-enriched genomic DNA library. Clones were then spotted onto gridded nylon filters and screened with 
radiolabelled SSR probes or subjected to enrichment with 'biotin labelled probes-streptavidin capture system' and sequenced. Cloning of DNA fragments prior to enrichment steps makes it ideal to screen for a wide range of SSR motifs and reduce/ avoid redundancy when compared to enrichment protocols. This method was successfully employed for the isolation of SSR markers from few crops like Citrus limon (Golein et al., 2006) and Phaseolus vulgaris (Blair et al., 2009).

\section{Utilization of PCR based molecular markers for generating} microsatellites

\section{SSRs derived from RAPD markers}

This method relies on the fingerprinting and subsequent blotting of the RAPD amplicons to nitrocellulose membrane, followed by the screening of positive clones by digoxygenin labelled probes and its detection by autoradiography (Random amplified hybridization microsatellites [RAHM]; Cifarelli et al., 1995). Some methods utilise labelled SSR probes and chemiluminescent system for the detection of SSRs (Ender et al., 1996) while others facilitate cloning and further screening of intense RAPD bands in duplicate colony PCR (with vector specific and repeat specific probes) for identifying SSR containing clones followed by direct sequencing [PCR isolation of microsatellite arrays (PIMA) approach; Lunt et al., 1999].

\section{SSRs derived from ISSR/SSR amplicons}

These methods are based on the fact that ISSR primers bind specifically to SSRs and facilitate the amplification of genomic DNA between two distinctly placed SSRs, providing an opportunity to design primers (based on the flanking regions) by cloning and sequencing of ISSR amplicons. The 'dual suppression method' (Lian et al., 2001; Lian and Hogetsu, 2002) involved cloning and sequencing of ISSR amplicons and initiating a nested PCR (with primers 'IP1'- designed from the region flanking the microsatellite sequence and 'IP2'- sequence between IP1 and the microsatellite sequence). Adaptor-ligated, restricted DNA libraries were then constructed using restricted DNA fragments ligated to a blunt ended suppression PCR adaptors ( $\mathrm{a}$ $48 \mathrm{mer}$ and a complementary $8 \mathrm{mer}$ capped with amino residue). Two adapter specific primers (AP1 and AP2), containing portions of the $48 \mathrm{mer}$ were designed and used for nested PCR amplification in two steps- first reaction with IP1 \& AP1 and second reaction using the PCR product as template and IP2 \& AP2 as primer. The single bands generated in the PCR were cloned and sequenced for designing a specific primer (IP3). A combination of either IP1 or IP2 primer along with the IP3 primer constituted the new SSR marker.

Another improved protocol described by Lian $e t$ al. (2006) differed from the earlier protocol in that a compound SSR primer and adapter primers were used for constructing genomic library. A specific primer (IP1) designed from the nucleotide sequence flanking the SSR and the initial compound SSR primer were used as a compound SSR marker.

Korpelainen et al. (2007) utilized genome screening with ISSR primers to obtain nucleotide sequence information flanking one side of the microsatellite followed by a restriction-ligation technique with a specific adaptor to facilitate sequence walking (in order to identify nucleotide sequences flanking the other side of the microsatellites). Wu et al. (2008) described an ICSSR (intercompound microsatellite) method in which DNA was amplified with a mixture of SSR primers and the products that showed multibands were cloned and sequenced. The primer designed from the flanking sequence and the SSR primer with shorter inner repeat constituted a compound SSR marker.

\section{SSRs developed using AFLP}

AFLP markers along with enrichment steps (Hakki and Akkaya, 2000; Zane et al., 2002) or a combination of randomly amplified microsatellite primer and a selective primer capable of amplifying restricted fragments containing SSR motifs (Van Eijk et al., 2001) were used for generating SSR markers.

Hakki and Akkaya (2000) utilised selectively amplified AFLP bands along with an enrichment step (using biotinylated target repeat oligonucleotide and streptavidin coated magnetic beads) to generate SSR markers. The enriched AFLP fragments were reamplified using same set of selective AFLP primer combinations, size selected, reamplified, and relatively long fragments (containing both restriction sites) were directly sequenced using site selective primers to reveal the nucleotide sequences flanking SSRs.

In the FIASCO (Fast Isolation by AFLP of Sequences Containing repeats) protocol, the AFLP bands were hybridized with biotinylated probes and subjected to selective capture using streptavidin-coated beads, followed by cloning and sequencing of enriched DNA fragments (Zane et al., 2002) to generate SSR markers.

Van Eijk et al. (2001) developed Microsatellite-AFLP (M-AFLP) technique that utilised the combination of a RAMP (Randomly Amplified Microsatellite Polymorphism) primer that binds to the microsatellite repeat at the transition point (between the repeat and flanking sequence) and a selective AFLP primer to amplify restriction fragments containing SSR motif sequences. The Amplified Fragment-Length Microsatellite (AFLM) approach (Douhan and Rizzo, 2003) utilised the selective amplification of the genomes with linker-adaptorPCR followed by enrichment of microsatellite motifs with 5' biotinylated oligonucleotides and recovery using streptavidin coated magnetic beads. The recovered fragments were re-amplified, cloned and sequenced to reveal flanking nucleotide sequence information.

\section{Other PCR based methods for SSR development}

Other methods utilised SAM (Selectively Amplified Microsatellite) for the selective amplification of restricted fragments with anchored primers, sequence tags, biotinylated probes and hybrid capture using streptavidin coated magnetic beads (Hayden and Sharp, 2001) or the use of multilocus marker system called RAMs (Random Amplified Microsatellites) to convert the amplified SSR bands to a marker, amplifying a single locus through a series of steps (Choy et al., 2005). The 
'Microsatellite Amplified Library' (MAL) approach (Acquadro et al., 2005) utilised a two step primer extension protocol that allowed the construction of enriched SSR libraries based on PCR and avoided the necessity of hybridisation, enrichment steps and conversion of SSRs into sequence tagged STMS markers.

Based on the cross-hybridisation of SSR sequences between distantly related organisms, Nolte et al. (2005) described a new protocol for the direct cloning of SSRs using an enrichment strategy. Wu et al. (2009) utilised a new genome walking method with a random tailed primer and multiple primer extensions (using Phu DNA polymerase) for isolating SSRs.

The 'Sequential Reverse Genome Walking' (SRGW) strategy (Joy et al., 2011) primarily involved the generation of a genomic walking (GW) library, which was enriched in two consecutive primary and nested secondary PCR steps (using SSR oligos as reverse primers in combination with two adaptor specific primers). The PCR products were cloned and sequenced. Based on the flanking sequence identified from one end of the microsatellite motif, two sets of flanking primers (F1 and nested secondary F2 primer) were designed. A 'sequential reverse walk' was then initiated with the rest of GW libraries using the flanking primers (F1 and F2) and the adaptor specific primers (AP1 and AP2). The secondary nested PCR products were cloned and sequenced to generate SSR markers.

The 'Recombinant microsatellite amplification' method (Wu et al., 2012) permitted rapid and large scale isolation of microsatellites by normalising adapter-ligated restricted DNA using a suppression PCR This was followed by the selective amplification of SSR containing sequences using anchored SSR primer and a suppressor primer. The amplified products were restricted, ligated, re-amplified using anchored primer, cloned and sequenced. From the sequenced clone, a primer targeting the SSR motif was designed while for designing the reverse primer, the whole SSR locus was isolated by genome walking.

\section{Using high throughput sequencing/ Next generation sequencing technology}

High throughput sequencing technology along with bioinformatics tools provide a superior alternative to the conventional methods used for developing SSR markers (Abdelkrim et al., 2009; Santana et al., 2009). With the advent of new next generation sequencing (NGS) platforms, large volumes of sequencing data are being generated that could be screened with the aid of bioinformatics tools for identifying microsatellite repeats. This avoids the construction of microsatelliteenriched DNA libraries and provides a rapid approach for the large-scale generation of microsatellite loci. The recent availability of massively parallel sequencing (MPS) facilitated the sequencing of microsatellite-enriched genomic libraries in multiplex pools, thereby reducing sample preparation and sequencing costs (Jennings et al., 2011). Current advances in NGS technology and reduction in sequencing costs will further enable easier, cheaper and rapid identification of microsatellite markers in future. The pyrosequencing technique has been applied for the generation of microsatellite markers from Comarum palustre (Somme et al., 2012), Vaccinium macrocarpon (Zhu et al., 2012), Linum usitatissimum etc. (Kale et al., 2012).

Using these various approaches, a large number of genomic SSR markers have been developed in several economically important crops. However, comparing the efficiency of SSR isolation protocols is generally difficult due to the difference in search criteria used for identifying SSRs and other variance factors existing among different laboratories and researchers (Techen $e t$ al., 2010). High redundancy, lack of SSRs in majority of sequenced clones and varying enrichment efficiency (observed when the same protocol is applied to members of same or different genus/ species) are some of the inherent problems associated with the improved protocols. Other factors that influence SSR frequencies include variation in sampling regions of the genome used for SSR detection (coding vs. non coding), library preparation and limits set for SSR detection using probe hybridization methods (Iniguez-Luy et al., 2008). High proportions of DNA fragments lacking microsatellite repeats in enrichment protocols is mainly attributed to the high level of non-specific binding of streptavidin coated magnetic beads to the DNA (St. John and Quinn, 2008). However, the variation in enrichment efficiency between different species arise mainly due to the quality of genomic DNA used for library construction, difference in genome size and complexity, variation in the frequency of microsatellite repeats in the genome and attrition problems during isolation.

\section{EST-SSR markers}

Large scale EST sequencing projects for gene discovery programmes have generated and deposited a wealth of EST sequences in databases (Rudd 2003). With the availability of SSR mining tools like TROLL (Castelo et al., 2002), MISA (Thiel et al., 2003), SciRoKo (Kofler et al., 2007), Msatcommander (Faircloth, 2008), QDD (Meglécz et al., 2010) etc., it has now become a fast approach to search for microsatellite repeats in the EST sequences/ databases and exploit the possibility of converting it into polymorphic SSR markers.

EST-SSR markers have both advantages and disadvantages. The generation of SSR markers from EST resources is relatively fast and inexpensive (Thiel et al., 2003; Gupta et al., 2003) and could be achieved rapidly using bioinformatics softwares (Varshney et al., 2005). EST-SSRs reveal variation in the expressed regions of the genome, thereby detecting perfect marker-trait associations (Gupta et al., 2003). They exhibit high transferability across a much broader taxonomic range (Gupta et al., 2003) and null alleles are less problematic (Leigh et al., 2003; Rungis et al., 2004) than those derived from untranslated regions (Rungis et al., 2004; Pashley et al., 2006).

However, the generation of EST-SSR markers is limited to the availability of EST sequences and hence 
restricted to economical and widely exploited crops (Varshney et al., 2005; Pashley et al., 2006), whose sequences are deposited or shared in the public domain. The relatively low abundance of SSRs within the transcribed region (Hancock, 1995; Katti et al., 2001) is also a limiting factor for the large scale development of genic SSR markers. Moreover, EST-SSR markers exhibit less polymorphism and are less efficient in distinguishing closely related individuals (Cho et al., 2000; Gupta et al., 2003; Chabane et al., 2005) because of greater DNA sequence conservation in transcribed or coding regions (Rungis et al., 2004; Varshney et al., 2005).

\section{Chloroplast SSR markers}

Microsatellites occurring in the chloroplast genome of higher plants (cpSSRs; Powell et al., 1995, 1996) are usually composed of mononucleotide ( $\mathrm{A}$ and $\mathrm{T}$ ) repeats rather than di-, tri- or tetra-nucleotide repeats (Bryan et al., 1999).

The identification and development of cpSSRs is achieved principally by utilizing the nucleotide sequence information retrieved from public databases (Weising and Gardner, 1999; Chung and Staub, 2003) and also by sequencing PCR products generated by "consensus/universal primers", capable of amplifying cpDNA regions in several species.

The de novo sequencing of noncoding chloroplast DNA regions is recommended to be the most efficient way for identifying large number of chloroplast microsatellites (Ebert and Peakall, 2009).

Taberlet et al. (1991) reported the first set of 'universal' PCR primers for analyzing intra-specific variation in the chloroplast nucleotide sequence across plant genera/ species. Later on, several universal primers were reported for amplifying chloroplast regions in various crops (Demesure et al., 1995; Dumolin-Lapegue et al., 1997; Weising and Gardner, 1999; Ebert and Peakall, 2009). Sequencing of amplicons generated by these universal cpDNA-PCR primers provide basic information about mononucleotide tracts and flanking sequences in the amplicons. Since the flanking regions of cpSSR loci are highly conserved, 'universal cPSSR primers' (Weising and Gardner, 1999; Chung and Staub, 2003; Ebert and Peakall, 2009) that enable the amplification of cpSSRs across species/taxa have been identified. Most of the molecular studies involving chloroplast microsatellites now rely on testing these universal cpSSRs in the target species.

Chloroplast SSR markers also have advantages and disadvantages. The haploid nature and high copy number of the chloroplast genome facilitate easy working of these markers using PCR based methods (Bryan et al., 1999). However, the mutation rate is lower than nuclear SSRs (Provan et al., 2001) and the level of polymorphism is variable across loci and species, with some loci found to be monomorphic in all species (Navascues and Emerson, 2005). The short length and limited number of alleles generated by cpSSRs further necessitate the confirmation of allele size by sequencing (Weising and Gardner, 1999).

\section{Cross-amplification/ transferability of microsatellite} markers

The high cost and labour involved in developing microsatellite repeats is a serious factor that restricts the wide-spread application of SSR markers in different plants. Hence the development of SSR markers is often focussed only to economically important crops. However, flanking sequences are reported to exhibit slower mutation rate than SSR region (Holmen et al., 2009), permitting their sequence conservation across species or genera. This homology allows the amplification of primers designed for one species to other members of the same species or genera (crossspecies/cross-genera amplification or transferability). Transferability offers potential for the low cost generation of microsatellite markers for related or distant species.

In general, the strategy is applicable to species belonging to the same genus or recently separated genera. However, successful cross-species amplification is inversely related to the evolutionary distance between two species (Primmer et al., 1996; Steinkellner et al., 1997), conservation of flanking sequences and maintenance of long arrays to generate sufficient polymorphisms (FitzSimmons et al., 1995). Among the class of SSR makers, EST-SSR markers shows greater cross-species transferability than genomic SSRs (Varshney et al., 2005), as they reside in the more conserved regions (genic) of the genome. Transferability studies are ample within genus (Roa et al., 2000; Takayama et al., 2008) and across genus (Raji et al., 2009; Datta et al., 2010).

\section{Conclusion}

It is evident that microsatellite markers are one of the most widely exploited molecular markers in various research areas, including the assessment of genetic diversity, gene mapping and marker assisted selection. Each type of SSR markers has its own advantages and disadvantages. Though the development of genomic SSR is cumbersome, it is of wide application in genetic diversity analysis and population genetics, due to its robustness and high polymorphism. EST-derived markers have a prominent role when the study is concerned with the identification of functional polymorphisms in key genes. Though several new isolation strategies have been described (Tab. 1), careful attempts need to be made to choose an appropriate strategy by considering factors like operation cost, rapid generation, high efficiency and species transferability. Among the methods available till date, when concerned with the short time and rapid mode of generation, NGS offers wide possibilities for the large scale generation of microsatellite markers. 
Fig.1. Strategies for the development of microsatellite markers

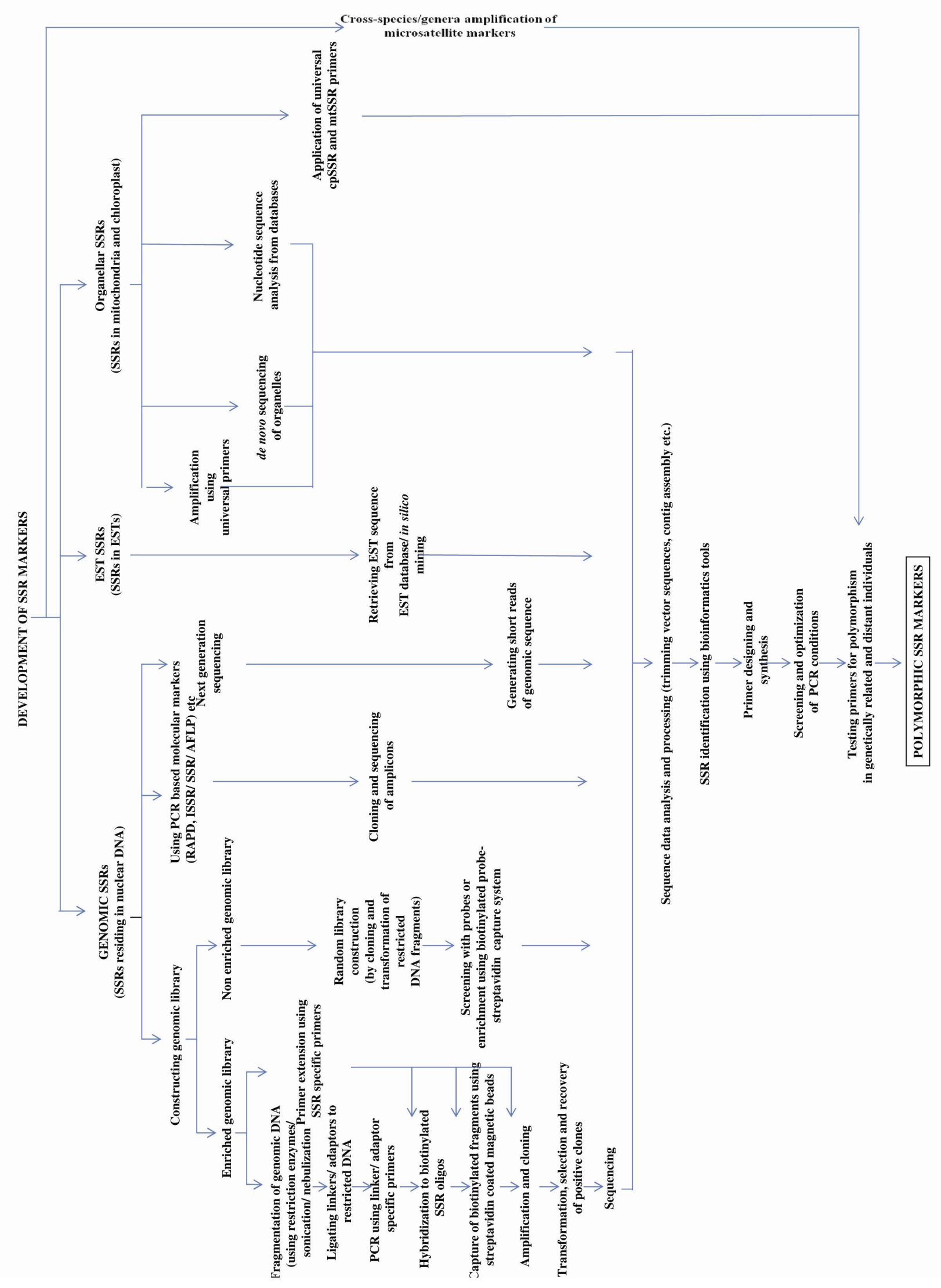


Tab. 1. Comparison of different methods used for the development of microsatellite markers

\begin{tabular}{|c|c|c|c|c|c|c|c|c|c|c|c|c|c|c|}
\hline \multirow[b]{2}{*}{ SSR type } & \multirow[b]{2}{*}{ Strategy } & \multirow[b]{2}{*}{ Method } & \multicolumn{8}{|c|}{ Methodology } & \multicolumn{4}{|c|}{ Advantages and Disadvantages } \\
\hline & & & $\begin{array}{c}\text { Restriction } \\
\text { digestion }\end{array}$ & $\begin{array}{l}\text { Adapter/ } \\
\text { linker } \\
\text { ligation }\end{array}$ & $\begin{array}{c}\text { PCR } \\
\text { amplification }\end{array}$ & $\begin{array}{l}\text { Enrichment } \\
\text { using probes/ } \\
\text { SSR primers }\end{array}$ & Cloning & Sequencing & $\begin{array}{c}\text { Primer } \\
\text { designing }\end{array}$ & $\begin{array}{l}\text { Testing of } \\
\text { primers }\end{array}$ & $\begin{array}{l}\text { Metholodology } \\
\text { used for } \\
\text { generation }\end{array}$ & $\begin{array}{c}\text { Cost } \\
\text { involved }\end{array}$ & $\begin{array}{l}\text { Time taken } \\
\text { for } \\
\text { development }\end{array}$ & $\begin{array}{l}\text { Labour } \\
\text { involved }\end{array}$ \\
\hline \multirow{8}{*}{$\begin{array}{l}\text { Genomic } \\
\text { SSR }\end{array}$} & \multirow{2}{*}{ Enrichment } & $\begin{array}{c}\text { Selective } \\
\text { hybridization }\end{array}$ & $\checkmark$ & $\checkmark$ & $\checkmark$ & $\checkmark$ & $\checkmark$ & $\checkmark$ & $\checkmark$ & $\checkmark$ & Complex & High & More & High \\
\hline & & $\begin{array}{c}\text { Primer } \\
\text { extension }\end{array}$ & & & $\checkmark$ & $\checkmark$ & $\checkmark$ & $\checkmark$ & $\checkmark$ & $\checkmark$ & Complex & High & More & High \\
\hline & Non-enrichment & & $\checkmark$ & $\checkmark$ & $\checkmark$ & & $\checkmark$ & $\checkmark$ & $\checkmark$ & $\checkmark$ & Complex & High & More & High \\
\hline & \multirow{4}{*}{$\begin{array}{c}\text { PCR based } \\
\text { molecular } \\
\text { markers }\end{array}$} & $\begin{array}{l}\text { RAPD } \\
\text { markers }\end{array}$ & & & $\checkmark$ & & $\checkmark$ & $\checkmark$ & $\checkmark$ & $\checkmark$ & Moderate & Moderate & Medium & Medium \\
\hline & & $\begin{array}{l}\text { ISSR/SSR } \\
\text { amplicons }\end{array}$ & & & $\checkmark$ & & $\checkmark$ & $\checkmark$ & $\checkmark$ & $\checkmark$ & Moderate & Moderate & Medium & Medium \\
\hline & & AFLP & $\checkmark$ & $\checkmark$ & $\checkmark$ & $\checkmark$ & $\checkmark$ & $\checkmark$ & $\checkmark$ & $\checkmark$ & Complex & High & More & High \\
\hline & & $\begin{array}{c}\text { Other } \\
\text { methods } \\
\text { (SLiM PCR, } \\
\text { Recombinant } \\
\text { microsatellite } \\
\text { amplification } \\
\text { etc) }\end{array}$ & $\checkmark$ & $\checkmark$ & $\checkmark$ & & $\checkmark$ & $\checkmark$ & $\checkmark$ & $\checkmark$ & Complex & High & Moderate & High \\
\hline & $\begin{array}{l}\text { High } \\
\text { throughput } \\
\text { /Next } \\
\text { generation } \\
\text { sequencing }\end{array}$ & & $*$ & $*$ & $*$ & & & $\checkmark$ & $\checkmark$ & $\checkmark$ & Easy & Medium & Less & Less \\
\hline EST-SSR & Data Mining & & & & & & & & $\checkmark$ & $\checkmark$ & Easy & Less & Less & Less \\
\hline cpSSR & $\begin{array}{l}\text { Data mining/ } \\
\text { Testing of } \\
\text { universal primers }\end{array}$ & & & & & & & & $\checkmark$ & $\checkmark$ & Easy & Less & Less & Less \\
\hline $\begin{array}{c}\text { Genomic } \\
\text { SSR/EST- } \\
\text { SSR/ cpSSR }\end{array}$ & $\begin{array}{l}\text { Transferability/ } \\
\text { Cross Species- } \\
\text { amplification }\end{array}$ & & & & & & & & & $\checkmark$ & Easy & Very less & Less & Less \\
\hline
\end{tabular}




\section{References}

Abdelkrim J, Robersten BC, Stanton JA, Gemmell NJ (2009). Fast, cost-effective development of species-specific microsatellite markers by genome sequencing. Biotechniques 46:185-191.

Acquadro A, Portis E, Albertini E, Lanteri S (2005). M-AFLPbased protocol for microsatellite loci isolation in Cynara cardunculus L. (Asteraceae). Mol Ecol Notes 5:272-274.

Bagshaw ATM, Pitt JPW, Gemmell NJ (2008). High frequency of microsatellites in $S$. cerevisiae meiotic recombination hotspots. BMC Genomics 9:49.

Bao S, Corke H, Sun M (2002). Microsatellites in starchsynthesizing genes in relation to starch physicochemical properties in waxy rice (Oryza sativa L.). Theor Appl Genet 105:898-905.

Blair MW, Torres MM, Pedraza F, Giraldo MC, Buendía HF, Hurtado N (2009). Development of microsatellite markers for common bean (Phaseolus vulgaris L.) based on screening of non-enriched, small-insert genomic libraries. Genome 52:772-82.

Brown J, Hardwick LJ, Wright AF (1995). A simple method for rapid isolation of microsatellites from yeast artificial chromosomes. Mol Cell Probe 9:53-57.

Bryan GJ, McNicol J, Ramsay G, Meyer RC, De-Jong WS (1999). Polymorphic simple sequence repeat markers in chloroplast genomes of solanaceous plants. Theor Appl Genet 99:859-867.

Castelo AT, Martins W, Gao GR (2002). TROLL-tandem repeat occurrence locator. Bioinformatics 18:634-636.

Chabane K, Ablett GA, Cordeiro GM, Valkoun J, Henry RJ (2005). EST versus genomic derived microsatellite markers for genotyping wild and cultivated barley. Genet Resour Crop Evol 52:903-909.

Chen H, Pulido JC, Duyk GM (1995). MATS: A rapid and efficient method for the development of microsatellite markers from YACs. Genomics 25:1-8.

Cho YG, Ishii T, Temnykh S, Chen X, Lipovich L, McCouch SR, Parl WD, Ayers N, Cartinhour S (2000). Diversity of microsatellites derived from genomic libraries and GenBank sequences in rice (Oryza sativa L.). Theor Appl Genet 100:713-722.

Choy CS, Siraj SS, Guan TS, Yusoff K (2005). Rapid and nonradioactive detection method of microsatellites in Mystus nemurus: A refined technique. Pertanika J Trop Agric Sci 28:73-77.

Chung SM, Staub JE (2003). The development and evaluation of consensus chloroplast primer pairs that posses highly variable sequence regions in diverse array of plant taxa. Theor Appl Genet 107:757-767.

Cifarelli RA, Gallitelli M, Cellini F (1995). Random amplified hybridization microsatellites (RAHM): isolation of a new class of microsatellite-containing DNA clones. Nucleic Acids Res 23:3802-3803.
Connell JP, Pammi S, Iqbal MJ, Huizinga T, Reddy AS (1998). A high throughput procedure for capturing microsatellites from complex plant genomes. Plant Mol Biol Rep 16:341-349.

Datta S, Mahfooz S, Singh P, Choudhary AK, Singh F, Kumar $S$ (2010). Cross-genera amplification of informative microsatellite markers from common bean and lentil for the assessment of genetic diversity in pigeonpea. Physiol Mol Biol Plants 16:123-134.

Demesure B, Sodzi N, Petit RJ (1995). A set of universal primers for amplification of polymorphic non-coding regions of mitochondrial and chloroplast DNA in plants. Mol Ecol 4:129-131.

Dixit A, Jin MH, Chung JW, Yu JW, Chung HK, Ma KH, Park YJ, Cho EG (2005). Development of polymorphic microsatellite markers in sesame (Sesamum indicum L.). Mol Ecol Notes 5:736-738.

Douhan GW, Rizzo DM (2003). Amplified Fragment Length Microsatellites (AFLM) might be used to develop microsatellite markers in organisms with limited amounts of DNA applied to Arbuscular Mycorrhizal (AM) fungi. Mycologia 95:368-373.

Dresselhaus T, Cordts S, Heuer S, Sauter M, Lörz H, Kranz E (1999). Novel ribosomal genes from maize are differentially expressed in the zygotic and somatic cell cycles. Mol Gen Genet 261:416-427.

Dumolin-Lapegue S, Pemonge MH, Petit RJ (1997). An enlarged set of consensus primers for the study of organelle DNA in plants. Mol Ecol 6:393-397.

Ebert D, Peakall R (2009). A new set of universal de novo sequencing primers for extensive coverage of noncoding chloroplast DNA: new opportunities for phylogenetic studies and cpSSR discovery. Mol Ecol Resour 9:777-783.

Edwards A, Civitello A, Hammond HA, Caskey CT (1991). DNA typing and genetic mapping with trimeric and tetrameric tandem repeats. Am J Hum Genet 49:746-756.

Edwards KJ, Barker JHA, Daly A, Jones C, Karp A (1996). Microsatellite libraries enriched for several microsatellite sequences in plants. Biotechniques 20:758.

Ellegren H (2004). Microsatellites: simple sequences with complex evolution. Nat Rev Genet 5:435-445.

Ender A, Schwenk K, Stadler T, Streit B, Schierwater B (1996) RAPD identification of microsatellites in Daphnia. Mol Ecol 5:437-441.

Estoup A, Jarne P, Cornuet JM (2002). Homoplasy and mutation model at microsatellite loci and their consequences for population genetics analysis. Mol Ecol 11:1591-1604.

Faircloth BC (2008). MSATCOMMANDER: detection of microsatellite repeat arrays and automated, locus-specific primer design. Mol Ecol Resour 8:92-94.

Fischer D, Bachmann K (1998). Microsatellite enrichment in organisms with large genomes (Allium cepa L.). BioTechniques 24:796-802. 
FitzSimmons NN, Moritz C, Moore SS (1995). Conservation and dynamics of microsatellite loci over 300 million years of marine turtle evolution. Mol Biol Evol 12:432-440.

Geng J, Li K, Zhang Y, Hu S (2010). A modified enrichment method to construct microsatellite library from plateau pika genome (Ochotona curzoniae). Genomics Proteomics Bioinform 8:72-76.

Gerber HP, Seipel K, Georgiev O, Hofferer M, Hug M, Rusconi S, Schaffner W (1994). Transcriptional activation modulated by homopolymeric glutamine and proline stretches. Science 263:808-811.

Glenn TC, Schable NA (2005). Isolating microsatellite DNA loci. Method Enzymol 395:202-222.

Golein B, Talaie A, Zamani Z, Moradi B (2006). Development and characterization of new microsatellite loci from lemon (Citrus limon). Int J Agr Biol 8:172-174.

Gupta PK, Balyan HS, Sharma PC, Ramesh B (1996). Microsatellites in plants: a new class of molecular markers. Curr Sci 70:45-54.

Gupta PK, Rustgi S, Sharma S, Singh R, Kumar N, Balyan HS (2003). Transferable EST-SSR markers for the study of polymorphism and genetic diversity in bread wheat. Mol Genet Genomics 270:315-323.

Hakki EE, Akkaya MS (2000). Microsatellite isolation using amplified fragment length polymorphism markers: no cloning, no screening. Mol Ecol 9:2152-2154.

Hamada HM, Petrino MG, Kakunaga T (1982). A novel repeated element with Z-DNA forming potential is widely found in evolutionarily diverse eukaryotic genomes. Proc Natl Acad Sci USA 79:6465-6469.

Hamilton MB, Pincus EL, Di-Fiore A, Fleischer RC (1999). Universal linker and ligation procedures for construction of genomic DNA libraries enriched for microsatellites. Biotechniques 27:500-507.

Hancock JM (1995). The contribution of slippage-like processes to genome evolution. J Mol Evol 41:1038-1047.

Hayden MJ, Sharp PJ (2001) Sequence tagged microsatellite profiling (STMP): a rapid technique for developing SSR markers. Nucleic Acids Res 29:e43.

He GH, Meng RH, Newman M, Gao GQ, Pittman RN, Prakash CS (2003). Microsatellites as DNA markers in cultivated peanut (Arachis hypogaea L.). BMC Plant Biol $3: 3$.

Holmen J, Vollestad LA, Jakobsen KS, Primmer CR (2009). Cross-species amplification of 36 cyprinid microsatellite loci in Phoxinus phoxinus (L.) and Scardinius erythrophthalmus (L.). BMC Res Notes 2:248.

Hulzink RJ, de Groot PF, Croes AF, Quaedvlieg W, Twell D, Wullems GJ, Van Herpen MM (2002). The 5'untranslated region of the ntp303 gene strongly enhances translation during pollen tube growth, but not during pollen maturation. Plant Physiol 129:342-353.

Iniguez-Luy F, Voort A, Osborn T (2008). Development of a set of public SSR markers derived from genomic sequence of a rapid cycling Brassica oleracea L. genotype. Theor Appl Genet 117:977-985.

Innan H, Terauchi R, Miyashita NT (1997). Microsatellite polymorphism in natural populations of the wild plant Arabidopsis thaliana. Genetics 146:1441-1452.

Jacob HJ, Lindpaintner K, Lincoln SE, Kusumi K, Bunker RK, Mao YP, Ganten D, Dzau VJ, Lander ES (1991). Genetic mapping of a gene causing hypertensive rat. Cell 67:213224.

Jennings TN, Knaus BJ, Mullins TD, Haig SM, Cronn RC (2011). Multiplexed microsatellite recovery using massively parallel sequencing. Mol Ecol Resourc. 11:1060-1067.

Joy N, Prasanth VP, Soniya EV (2011). Microsatellite based analysis of genetic diversity of popular black pepper genotypes in South India. Genetica 139:1033-1043.

Kale SM, Pardeshi VC, Kadoo NY, Ghorpade PB, Jana MM, Gupta VS (2012). Development of genomic simple sequence repeat markers for linseed using next-generation sequencing technology. Mol Breeding Doi:10.1007/s11032-011-9648-9.

Kalia RK, Rai MK, Kalia S, Singh R, Dhawan AK (2011). Microsatellite markers: an overview of the recent progress in plants. Euphytica 177:309-334.

Kandpal RP, Kandpal G, Weissman SM (1994). Construction of libraries enriched for sequence repeats and jumping clones, and hybridization selection for region-specific markers. Proc Natl Acad Sci USA 91:88-92.

Karagyozov L, Kalcheva ID, Chapman VM (1993). Construction of random small-insert genomic libraries highly enriched for simple sequence repeats. Nucleic Acids Res 21:3911-3912.

Kashi Y, King D, Soller M (1997). Simple sequence repeats as a source of quantitative genetic variation. Trends Genet 13:74-78.

Katti MV, Ranjekar PK, Gupta VS (2001). Differential distribution of simple sequence repeats in eukaryotic genome sequences. Mol Biol Evol 18:1161-1167.

Kindiger B (2006) Cross-species amplification of Lolium microsatellites in Poa ssp. Grassland Sci 52:105-115.

Kofler R, Schlotterer C, Lelley T (2007). SciRoKo: a new tool for whole genome microsatellite search and investigation. Bioinformatics 23:1683-1685.

Kooiker M, Airoldi CA, Losa A, Manzotti PS, Finzi L, Kater MM, Colombo L (2005). BASIC PENTACYSTEINE1, a GA binding protein that induces conformational changes in the regulatory region of the homeotic Arabidopsis gene SEEDSTICK. Plant Cell 17:722-729.

Korpelainen H, Kostamo K, Virtanen V (2007). Microsatellite marker identification using genome screening and restriction-ligation. Biotechniques 42:479-486.

Kumar P, Gupta VK, Misra AK, Modi DR, Pandey BK (2009). Potential of molecular markers in plant biotechnology. Plant Omics J 2:141-162

Kumpatla SP, Manley MK, Horne EC, Gupta M, Thompson SA (2004). An improved enrichment procedure to develop 
11

multiple repeat classes of cotton microsatellite markers. Plant Mol Biol Rep 22:85a-85i.

Lagercrantz U, Ellegren H, Andersson L (1993). The abundance of various polymorphic microsatellite motifs differs between plants and vertebrates. Nucleic Acids Res 21:1111-1115.

Lawson MJ, Zhang L (2006). Distinct patterns of SSR distribution in the Arabidopsis thaliana and rice genomes. Genome Biol 7:R14.

Lee JM, Nahm SH, Kim YM, Kim BD (2004). Characterization and molecular genetic mapping of microsatellite loci in pepper. Theor Appl Genet 108:619627.

Leigh F, Lea V, Law J, Wolters P, Powell W, Donini P (2003). Assessment of EST- and genomic microsatellite markers for variety discrimination and genetic diversity studies in wheat. Euphytica 133:359-366.

Levinson G, Gutman GA (1987). Slipped-strand mispairing: a major mechanism for DNA sequence evolution. Mol Biol Evol 4:203-221.

Li YC, Korol AB, Fahima T, Beiles A, Nevo E (2002). Microsatellites: genomic distribution, putative functions and mutational mechanisms: a review. Mol Ecol 11:24532465.

Lian C, Hogetsu T (2002). Development of microsatellite markers in black locust (Robinia pseudoacacia) using a dualsuppression-PCR technique. Mol Ecol Notes 2:211-213.

Lian CL, Wadud MA, Geng QF, Shimatani K, Hogetsu T (2006). An improved technique for isolating codominant compound microsatellite markers. J Plant Res 119:415417.

Lian CL, Zhou ZH, Hogetsu T (2001). A simple method for developing microsatellite markers using amplified fragments of inter-simple sequence repeat (ISSR). J Plant Res 114:381-385.

Litt M, Luty JA (1989). A hypervariable microsatellite revealed by in vitro amplification of a dinucleotide repeat within the cardiac muscle actin gene. Am J Hum Genet 44:397-401.

Lunt DH, Hutchinson WF, Carvalho GR (1999). An efficient method for PCR-based identification of microsatellite arrays (PIMA). Mol Ecol 8:893-894

Martin P, Makepeace K, Hill SA, Hood DW, Moxon ER (2004). Microsatellite instability regulates transcription factor binding and gene expression. Proc Natl Acad Sci USA 102:3800-3804.

McConnell R, Middlemist S, Scala C, Strassman JE, Queller DC (2007). An unusually low microsatellite mutation rate in Dictyostelium discoideum, an organism with unusually abundant microsatellites. Genetics 177:1499-1507.

Meglécz E, Costedoat C, Dubut V, Gilles A, Malausa T, Pech N, Martin JF (2010). QDD: a user-friendly program to select microsatellite markers and design primers from large sequencing projects. Bioinformatics 26:403-404.

Meister RJ, Williams LA, Monfared MM, Gallagher TL, Kraft
EA, Nelson CG, Gasser CS (2004). Definition and interactions of a positive regulatory element of the Arabidopsis INNER NO OUTER promoter. Plant J 37:426-438

Metzgar D, Bytof J, Wills C (2000). Selection against frameshift mutations limits microsatellite expansion in coding DNA. Genome Res 10:72-80.

Morgante M, Hanafey M, Powell W (2002). Microsatellites are preferentially associated with nonrepetitive DNA in plant genomes. Nature Genet 30:194-200.

Navascués M, Emerson BC (2005). Chloroplast microsatellites: measures of genetic diversity and the effect of homoplasy. Mol Ecol 14:1333-1341.

Nolte AW, Stemshorn KC, Tautz D (2005). Direct cloning of microsatellite loci from Cottus gobio through a simplified enrichment procedure. Mol Ecol Notes 5:628-636.

Nunome T, Negoro S, Miyatake K, Yamaguchi H, Fukuoka H (2006). A protocol for the construction of microsatellite enriched genomic library. Plant Mol Biol Rep 24:305-312.

Oliveira EJ, Paidua JG, Zucchi MI, Vencovsky R, Vieira MLC (2006). Origin, evolution and genome distribution of microsatellites. Genet Mol Res 29:294-307.

Ostrander EA, Jong PM, Rine J, Duyk G (1992). Construction of small-insert genomic DNA libraries highly enriched for microsatellite repeat sequences. Proc Natl Acad Sci USA 89:3419-3423.

Paetkau D (1999). Microsatellites obtained using strand extension: An enrichment protocol. Biotechniques 26:690697.

Pandolfo M (1992). A rapid method to isolate (GT)n repeats from yeast artificial chromosomes. Nucleic Acids Res 20:1154.

Parida SK, Kalia SK, Sunita K, Dalal V, Hemaprabha G, Selvi, A, Pandit A, Singh A, Gaikwad K, Sharma TR, Srivastava PS, Singh NK, Mohapatra T (2009). Informative genomic microsatellite markers for efficient genotyping applications in sugarcane. Theor Appl Genet 118:327-338.

Pashley CH, Ellis JR, McCauley DE, Burke JM (2006). EST databases as a source for molecular markers: lessons from Helianthus. J Hered 97:381-388.

Pemberton JM, Slate J, Bancroft DR, Barrett JA (1995). Nonamplifying alleles at microsatellite loci: a caution for parentage and population studies. Mol Ecol 7:249-252.

Perutz MF, Johnson T, Suzuki M, Finch JT (1994). Glutamine repeats as a polar zippers: their possible role in inherited neurodegenerative diseases. Proc Natl Acad Sci USA 91:5355-5358.

Powell W, Machray GC, Provan J (1996). Polymorphism revealed by simple sequence repeats. Trends Plant Sci $1: 215-222$.

Powell W, Morgante M, Andre C, McNicol JW, Machray GC, Doy Tingey SV, Rafalski JA (1995). Hypervariable microsatellites provide a general source of polymorphic DNA markers for the chloroplast genome. Curr Biol 5:215-222. 
Primmer CR, Moller AP, Ellegren H (1996). A wide-range survey of cross-species microsatellite amplification in birds. Mol Ecol 5:365-378.

Prochazka M (1996). Microsatellite hybrid capture technique for simultaneous isolation of various STR markers. Genome Res 6:646-649.

Provan J, Powell W, Hollingsworth PM (2001). Chloroplast microsatellites: new tools for studies in plant ecology and evolution. Trends Ecol Evol 16: 142-147.

Raji AAJ, Anderson JV, Kolade OA, Ugwu CD, Dixon AGO, Ingelbrecht IL (2009). Gene-based microsatellites for cassava (Manihot esculenta Crantz): prevalence, polymorphisms, and cross-taxa utility. BMC Plant Biol 9:118.

Rassmann K, Schlotterer C, Tautz D (1991). Isolation of simple sequence loci for use in polymerase chain reactionbased DNA fingerprinting. Electrophoresis 12:113-118.

Refseth UH, Fangan BM, Jakobsen KS (1997). Hybridization capture of microsatellites directly from genomic DNA. Electrophoresis 18:1519-1523.

Roa AC, Chavarriaga-Aguirre P, Duque MC, Maya MM, Bonierbale MW, Iglesias C, Tohme J (2000). Crossspecies amplification of cassava (Manihot esculenta) (Euphorbiaceae) microsatellites: allelic polymorphism and degree of relationship. Am J Bot 87:1647-1655.

Robic A, Dubois C, Milan D, Gellin J (1994). A rapid method to isolate microsatellite markers from cosmid clones. Mamm Genome 5:177-179.

Rose O, Falush D (1998). A threshold size for microsatellite expansion. Mol Biol Evol 15:613-615.

Rudd S (2003). Expressed sequence tags: alternative or complement to whole genome sequences? Trends Plant Sci 8:321-329.

Rungis D, Berube Y, Zhang J, Ralph S, Ritland CE, Ellis BE, Douglas C, Bohlmann, J, Ritland K (2004). Robust simple sequence repeats markers for spruce (Picea spp.) from expressed sequence tags. Theor Appl Genet 109:12831294.

Santana QC, Coetzee MPA, Steenkamp ET, Mlonyeni OX, Hammond GNA, Wingfield MJ, Wingfield BD (2009). Microsatellite discovery by deep sequencing of enriched genomic libraries. Biotechniques 46:217-223.

Schlotterer C, Tautz D (1992). Slippage synthesis of simple sequence DNA. Nucleic Acids Res 20:211-215.

Selkoe KA, Toonen RJ (2006). Microsatellites for ecologists: A practical guide to using and evaluating microsatellite markers. Ecol Lett 9:615-629.

Somme L, Raabova J, Jacquemart AL, Raspe O (2012). Development and multiplexing of microsatellite markers using pyrosequencing in the clonal plant Comarum palustre (Rosaceae). Mol Ecol Resour 12:91-97.

Squirrell J, Hollingsworth PM, Woodhead M, Russell J, Lowe AJ, Gibby M, Powell W (2003) How much effort is required to isolate nuclear microsatellites from plants? Mol
Ecol 12:1339-1348.

St John J, Quinn TW (2008). Rapid capture of DNA targets. Biotechniques 44:259-264.

Steinkellner H, Lexer C, Turetschek E, Glössl J (1997). Conservation of (GA)n microsatellite between Quercus species. Mol Ecol 6:1189-1194.

Stepien L, Mohler V, Bocianowski J, Koczyk G (2007). Assessing genetic diversity of Polish wheat (Triticum aestivum) varieties using microsatellite markers. Genet Resour Crop Evol 54, 1499-1506.

Taberlet P, Gielly L, Pautou G, Bouvet J (1991). Universal primers for amplification of three noncoding regions of chloroplast DNA. Plant Mol Biol 17:1105-1109.

Takayama K, Tamura M, Tateishi Y, Kajita T (2008). Isolation and characterization of microsatellite loci in the red mangrove Rhizophora mangle (Rhizophoraceae) and its related species. Conserv Genet 9:1323-1325.

Tautz D (1989). Hypervariability of simple sequences as a general source for polymorphic DNA markers. Nucleic Acids Res 17:6463-6471.

Tautz D, Renz M (1984). Simple sequence repeats are ubiquitous repetitive components of eukaryotic genomes. Nucleic Acids Res 12:4127-4138.

Techen N, Arias RS, Glynn NC, Pan Z, Khan IA, Scheffler BE (2010). Optimized construction of microsatellite-enriched libraries. Mol Ecol Resour 10:508-515.

Temnykh S, DeClerck G, Lukashova A, Lipovich L, Cartinhour S, McCouch S (2001). Computational and experimental analysis of microsatellites in rice (Oryza sativa L.): Frequency, length variation, transposon associations, and genetic marker potential. Genome Res 11:1441-1452.

Thiel T, Michalek W, Varshney RK, Graner A (2003). Exploiting EST databases for the development and characterization of gene-derived SSR-markers in barley (Hordeum vulgare L.). Theor Appl Genet 106:411-422.

Toth G, Gaspari Z, Jurka J (2000). Microsatellites in different eukaryotic genomes: survey and analysis. Genome Res 10:967-981.

Van-Eijk M, De-Ruiter M, Broekhof J, Peleman J (2001). Discovery and detection of polymorphic microsatellites by microsatellite- AFLP. In: Plant and Animal Genome IX Abstracts (ed. Heller SR), p. 143. Scherago International, New York.

Varshney RK, Graner A, Sorrells ME (2005). Genic microsatellite markers in plants: features and applications. Trends Biotechnol 23:48-55.

Weber JL, May PE (1989) Abundant class of human DNA polymorphisms which can be typed using the polymerase chain reaction. Am J Hum Genet 44:388-396.

Weising K, Gardner RC (1999). A set of conserved PCR primers for the analysis of simple sequence repeat polymorphisms in chloroplast genomes of dicotyledonous angiosperms. Genome 42:9-19. 
13

White G, Powell W (1997). Isolation and characterization of microsatellite loci in Swietenia humilis (Meliaceae): an endangered tropical hardwood species. Mol Ecol 6:851860.

Wolko Ł, Antkowiak W, Lenartowicz E, Bocianowski J (2010). Genetic diversity of European pear cultivars (Pyrus communis L.) and wild pear (Pyrus pyraster (L.) Burgsd.) inferred from microsatellite markers analysis. Genet Resour Crop Evol 57:801-806.

Wu B, Kurokochi H, Hogetsu T (2009). Development of 12 microsatellite markers in Euptelea polyandra by a random tailed genome-walking method using Phi29 DNA polymerase. Conserv Genet Resour 1:59-61.

Wu B, Lu P, Zhang Z (2012). Recombinant microsatellite amplification: a rapid method for developing simple sequence repeat marker. Mol Breeding 29:53-59.
Wu B, Shiokawa A, Matsushita N, Hogetsu T (2008). Isolation of compound microsatellite loci in the herbaceous perennial Cirsium purpuratum (Maxim.) Matsum. Mol Ecol Resour 8:814-817.

Zane L, Bargelloni L, Patarnello T (2002). Strategies for microsatellite isolation: a review. Mol Ecol 11:1-16.

Zhang LD, Yuan DJ, Yu SW, Li ZG, Cao YF, Miao ZQ, Qian HM, Tang KX (2004). Preference of simple sequence repeats in coding and non-coding regions of Arabidopsis thaliana. Bioinformatics 20:1081-1086

Zhu H, Senalik D, McCown BH, Zeldin EL, Speers J, Hyman J, Bassil N, Hummer K, Simon PW, Zalapa JE (2012). Mining and validation of pyrosequenced simple sequence repeats (SSRs) from American cranberry (Vaccinium macrocarpon Ait.). Theor Appl Genet 124:87-96. 